\title{
Workplace Wellness Champions: Lessons Learned and Implications for Future Programming
}

\author{
Megan Amaya, PhD, CHES \\ Bernadette Mazurek Melnyk, PhD, RN, CPNP/PMHNP, FAANP, FNAP, FAAN \\ Brenda Buffington, EdD \\ Lauren Battista, MPH \\ The Ohio State University
}

\begin{abstract}
Background: Wellness champion teams can be a critical "grass roots" strategy in building a culture of worksite wellness; however, little is known about key elements of programs to prepare individuals for this role and their level of impact.

Aim: 'To describe the components of a worksite wellness champion program at a large public land grant university in the Midwest and the characteristics of individuals who participate in this role.

Methods: The Wellness Innovator program components, including processes of recruitment and retention, as well as demographic data of the Innovators are described.

Results: 464 Innovators currently serve in the role. Support from supervisors/managers is key for sustained Innovator engagement.

Conclusions: The Wellness Innovator program is an important strategy in encouraging faculty and staff to participate in wellness activities and services. More research is needed to determine the impact of wellness champion teams on health and wellness outcomes.
\end{abstract}

Submitted 24 February 2017; accepted 29 March 2017

Keywords: worksite, wellness, champions, health promotion, workplace, leadership support

It has been well documented that unhealthy lifestyle behaviors contribute to poor health outcomes, increased healthcare costs, loss of productivity, and problems with absenteeism (Borah, Egginton, \& Shah, 2015; Claxton, Rae, \& Panchal, 2011; James, 2013). Many worksites are investing in workplace health promotion programs to counteract those outcomes. There are many facets to a successful workplace health promotion program, including but not limited to: (1) leadership support, (2) health education and behavior change programs, (3) environmental and cultural supports, (4) management of chronic conditions, (5) health policies, 6) wellness champion networks, and (6) aligning healthy lifestyle behaviors and health outcomes with benefits. Arguably, all are important components in a comprehensive wellness program.

Practitioners in the field of worksite health promotion strongly acknowledge the need for the "grass roots" efforts. "Grass roots" refers to employees who are not necessarily experts in the field of health and wellness, but have a passion for it, personally and professionally, in the sense they want to promote wellness among their colleagues. Practitioners in the field of workplace health promotion refer to these grass roots employees as wellness champions, wellness ambassadors, and wellness mentors, to name a few. Spoonheim and Pronk (2016) explain that wellness champions appear at every level of a company and often are intrinsically motivated to take on the role of being a champion. Whether formally or informally assigned to this role, champions are considered an important social support to others and have a powerful voice among all employees (Spoonheim \& Pronk, 2016). It has been noted that peers can have a powerful influence on health behaviors and outcomes (Borah et al, 2015). The social support provided by individuals has the potential to improve the health and wellness of peers within their social networks (Borah et al., 2015). Linnan, Fisher and Hood (2013) recommend that champions "should have the right blend of encouragement, nurturing, enthusiasm, passion, and dedication to the task, as well as the ability to tailor his or her approach to the employee while maintaining an appropriate level of respect, privacy and space." This peer support approach does not encourage mandatory peer support assignments, but rather peer supporters that are encouraged and allowed to volunteer and serve. Spoonheim \& Pronk (2016) note the importance of a multilevel leadership approach (the wellness champion network), ensuring that employees from all levels are heard, and bottom-up efforts 
are both recognized and nurtured.

Several studies have documented the impact that social ties can have on our health (Aoun, Sainsbury, Mullan, \& Shahid, 2017; Christakis \& Fowler, 2007; McPherson, Joseph, \& Sullivan, 2004; Leahey \& Wing, 2012; Locock, Dopson, Chambers, \& Gabbay, 2001). A study by Escoffery, Kegler, and Alcantara (2011) found that naturally occurring support from workplace peers was important in encouraging changes in eating and weight among employees in small, rural worksites in Georgia. During the in-depth interviews, coworkers were acknowledged for providing helpful information and encouragement about healthy eating, including the types of foods individuals eat as well as methods for eating healthy, providing input on food preparation, and discouraging each other from eating unhealthy foods. Several studies have specifically focused on understanding the role of peer support in health promotion at worksites and how coworker support influences employees' diet and nutrition behaviors. In a 16-month randomized controlled study involving 26 worksites, peer health advisors at each intervention worksite were trained to serve as role models for breast and cervical cancer screening behaviors to female employees at their worksites (Allen, Stoddard, Mays, \& Sorensen, 2001). The peer health advisors were responsible for disseminating breast and cervical cancer information to employees, providing social support, and facilitating the processes of screening becoming a social norm in the workplace. Positive results were observed among worksite participants in the intervention condition; participants had a significantly greater cervical cancer screening rate (odds ratio $[\mathrm{OR}]=1.28,95 \%$ confidence interval $=1.01,1.62$ ) than did comparison group participants. Worksite peer support has also had a positive influence on substance abuse prevention behaviors. Bondy and Bercovitz (2013) conducted a qualitative content analysis of an existing Internetbased forum for individuals working in the construction and renovation sector. Within the forum, peer support was evident for encouraging smoking cessation, a highly prevalent cancer risk behavior among individuals in this industry. In particular, the researchers observed that peer support was provided for avoiding smoking, and focused on skills for smoking cessation, personal commitment, and the benefits associated with smoking cessation. Closely related to these findings, results of a systematic review of worksite interventions for alcohol problems conducted by Webb, Shakeshaft, Sanson-Fisher, and Harvard (2009) suggest that peer support may be effective for addressing alcohol abuse.

In order to positively impact the health and wellness of employees within their organization, teams of wellness champions not only have to participate in a wellness champion program, but must remain consistently and highly engaged. The issue of sustainable engagement is a challenge because, in most cases, it is a volunteer role. Because employees are primarily responsible for accomplishing their assigned role responsibilities, it is important that wellness champions that volunteer for the role are highly committed and have their manager's support. To date, few publications exist regarding the impact of wellness champions. In a recent study conducted at the Mayo Clinic, findings indicated that the rate of overall health and wellness was higher in employees familiar with the Wellness Champion Program compared to those not familiar with the wellness champion program, and participants were more likely to agree that the organization facilitated a supportive environment to live a healthy lifestyle (Wieneke, Clark, \& Olsen, 2016).

Before the program facilitators can establish effectiveness of the wellness innovator team, it is important to understand how the level of engagement in the team affects the success of the program. At The Ohio State University, facilitators of the Wellness Champion Program are implementing a study to determine the answers to these questions, which will be addressed later in this article. However, the main purpose of this paper is to describe the specific components of the wellness champion program, entitled the wellness innovator program, at a large public land grant academic institution that houses an academic medical center and describe the individuals who participate in it.

\section{PROGRAM METHODS}

The team of wellness champions at The Ohio State University is called the Buckeye Wellness Innovators. The program is implemented out of the Office of the Vice President for Health Promotion and Chief Wellness Officer (CWO) and co-facilitated by the Director of Health Promotion and Wellness Program Manager. The Innovator program began in January of 2012. There are 464 Wellness Innovators across the university, representing 160 academic and medical center departments/units. 


\section{Roles and Responsibilities}

Buckeye Wellness Innovators are University and medical center faculty and staff who have a special interest in facilitating a culture and environment of wellness in their college, unit, or department. They champion universitywide wellness initiatives in their departments and advance the One University Health and Wellness strategic plan. Innovators are responsible for communicating health and wellness activities to their colleagues, encouraging and motivating faculty and staff to participate in wellness initiatives, engage in healthy lifestyle practices, utilize wellness resources, and plan and conduct wellness activities in their colleges, departments or units. They are required to devote two to three hours per month to the program and to coordinate their efforts with the co-facilitators and human resources personnel when applicable to ensure health and wellness communications are aligned. Innovators who are faculty at the university have the opportunity to count the Innovator role towards their service requirements.

\section{One University Approach to Wellness}

The vision of the wellness program at Ohio State is: "To be the healthiest university on the globe." To achieve this ambitious vision, the One University Health and Wellness Council provides strategic leadership and direction for health and wellness initiatives across the university. The council is led by the University's Vice President for Health Promotion and Chief Wellness Officer and is comprised of key leaders across the University who have responsibility for various aspects of health and wellness for faculty, staff and students along with representation from faculty, staff, students, and University communications. The University embraces nine dimensions of wellness and uses this comprehensive model as a guide for designing, implementing, and evaluating wellness programs and services for students, faculty and staff based on the socioecological framework to guide evidence-based intervention and outcomes assessment (Melnyk, Amaya, Szakacha, \& Hoying, 2016). Innovators promote wellness programs and resources designed to enhance faculty and staff wellness among all nine dimensions throughout the calendar year. In addition, the socio-ecological framework adopted by the University represents a vital piece of its health promotion efforts, targeting multiple levels of impact: individual, social networks and family, culture and environment, and policy. Oftentimes, when Innovators plan wellness activities for their department, they address one or more of the levels mentioned above.

\section{Recruitment}

To recruit new Wellness Innovators, the program facilitators disseminate a call for Innovators three times per year. Recruitment information is sent out through a series of emails: From the daily internal university system to all faculty and staff; from the CWO to University deans and vice presidents (VP); to human resource professionals in colleges and VP units; and finally, to current Wellness Innovators and individuals who have participated in University health and wellness programs. Wellness Innovators are self-nominated or are nominated by a manager/supervisor or existing Wellness Innovator for this volunteer role. Innovators must have approval from their supervisor to participate in the program.

Once recruited, new Innovators attend a four-hour orientation. At this orientation, new Innovators are introduced to the University's wellness vision, mission and core goals. They also review the wellness program components applicable to the faculty and staff, educational health promotion offerings, wellness services and the employee assistance program. They then receive a detailed description of their new responsibilities. Following the orientation, program facilitators contact the new Innovators to schedule a one-hour strategy session. The program facilitators outline role expectations and work with the Innovators to construct a plan for their department or unit over the next 12 months. The Innovator's manager/supervisor is invited to the meeting to ensure their direct leader is aware of the responsibilities and expected time commitment of being an Innovator and is supportive of their role. In early 2017, the program facilitators implemented a new retention effort, which includes meeting with each Innovator on

an annual basis to discuss successes and challenges to their role. Please see Figure 1 for a breakdown of Innovator on-boarding and roles/responsibilities throughout the calendar year. 


\section{Demographics of the Innovators}

Currently, there are 464 Innovators at The Ohio State University, 224 represent the medical center and 240 represent the academic side of the university. The majority of Innovators are University staff (447) and 15 are faculty. Innovators are located at all five regional campuses of the university and ten work in Ohio's county positions within the University's extension offices. A majority of Innovators are female (over $85 \%$ ).

\section{Strategy Sessions}

Following the orientation, the program facilitators meet with the Innovator or Innovators if there is more than one in a department, for a strategy session. Their discussion includes the following areas:

1. The environment of the department: Manager support, perceptions of stress levels, perceived interest among colleagues in engaging in healthy behaviors, and demographics. The Innovator has the option to conduct a short baseline survey, asking colleagues which areas of wellness are of most interest.

2. Overall adopted university wellness strategies.

3. A description of their main responsibilities (communication, encouragement, and planning activities), and expectations. A checklist of annual activities is given to the innovators during the meeting for tracking their activities and efforts.

4. Examples of what other Innovators are implementing.

5. Funding opportunities for supporting department/unit-specific wellness efforts.

6. Collaborating with human resources where appropriate.

7. Communication resources.

8. Incentives and benefits that are exclusively available to them such as complimentary participation in the Health Athlete corporate program.

9. Action items for follow-up.

The session takes approximately one hour. Program facilitators follow-up with the Innovators throughout the year to provide ongoing support.

\section{Financial Support through Funding Opportunities}

Innovators are invited to apply for grants, up to $\$ 500$, to support the on-going efforts to influence the wellness culture and environment of their department/unit. This biannual funding opportunity encourages Innovators to submit a proposal to fund activities or programs that promote physical activity, healthy eating or emotional well-being among their colleagues. Thus far, 30 Innovators have received grants to support their proposals. The program facilitators require a written report one (1) year from the date of implementation. Innovators are required to track participation, and are highly encouraged to report implementation details and outcomes data.

\section{Strategic Communication}

Communication is an important aspect of the Wellness Innovators program; however, efforts need to be strategic. Once a month, the co-facilitator of the program sends out an email with a couple of high priority health and wellness announcements. The second co-facilitator creates a monthly email newsletter that Innovators can share with their departments. In addition, the private Facebook group allows Innovators to share ideas, pictures, activities and resources. The program facilitators also contribute content to the page. Twice a year, a webinar is held to update 
Innovators on current and upcoming events. Biannual luncheons spotlight Innovators' efforts, provide opportunities for brainstorming ways to overcome challenges, and host motivational guest speakers to re-energize the group.

\section{Unique Opportunities}

Innovators have the benefit of attending a free two-day energy management course as a part of their role and are highly encouraged to utilize this benefit. The energy management course, called Health Athlete, mirrors the Human Performance Institute's Corporate Athlete program (2011), and is part of a formal partnership with Johnson \& Johnson, owners of the Corporate Athlete program. Not only does the Health Athlete program help develop their personal energy management skills but allows them to take back some strategies for achieving high levels of wellbeing to their departments and colleagues.

\section{Manager and Supervisor Support}

In 2016, the health promotion experts at Ohio State began efforts to engage mid-level managers and supervisors in the University's health and wellness initiative. This effort is currently ongoing. Managers and supervisors are essential to the success of the overall program because they provide support and encouragement to those that work for them (i.e., a manager allowing staff to attend a wellness fair on work time, or encouraging staff to take 15 minutes during their work hours to get a biometric screening). We have noted that if the Innovator's manager/supervisor does not support their employee to engage in worksite wellness activities, the wellness efforts in that department are likely to stall. When the manager/supervisor does not receive the support that is needed to fulfill their role, they tend to become disengaged from the program. Therefore, the facilitators of the program ask all Innovators to obtain approval from their direct supervisor before entering the program. Managers/supervisors also are invited to attend the one-hour strategy session as well as some or all of the orientation so that they are aware of the responsibilities and expected time commitment of the Innovator role and can provide support. In the event that a supervisor does not grant permission for their employee to engage in the Innovator program, the facilitators of the program provide the manager with additional information, such as the University president's wellness goal, to further increase her/his understanding. This is generally received well and permission is granted.

\section{Activities Being Conducted by the Innovators}

The program facilitators instruct Innovators to create and implement activities that focus on one or more of the nine dimensions of wellness. Innovators promote several areas of wellness, including: Healthy eating, physical activity and stress management/coping, but are able to promote wellness in other dimensions, such as financial, social, spiritual, environmental, occupational and intellectual. With 464 Innovators enrolled in the program, a wide variety of health and wellness activities are implemented within their departments/units. For example, Innovators often plan healthy food demonstrations and potlucks, lead teams for physical activity competitions, and encourage stairwell usage. Many Innovators enlist health promotion experts, such as members of the Chief Wellness Officer's wellness team or the university's health plan health coaches, to present on wellness topics. These presentations are often done during the lunch hour or in staff and faculty meetings. Innovators plan yoga and other fitness classes in their departments/ units, and work with the program facilitators, colleagues, university health and wellness service departments, and at times, local businesses to secure instructors and space. Additionally, Innovators are provided with toolkits, such as the American Heart Association's Healthy Workplace Food and Beverage Toolkit (American Heart Association, 2015), which enables them to use evidence-based information when selecting healthy food options for work-related meetings and functions.

\section{Assessing Engagement and Impact}

To date in the literature, few studies have been conducted to assess the level of engagement necessary and impact of wellness champion teams on health and wellness outcomes (Weineke et al., 2015). In order to further the field 
and provide important information to leadership about the important benefits of the Innovators, efforts are being made to document important outcomes of the Innovator program at Ohio State University. Currently, an IRB approved study is being conducted to measure the actual engagement of those enrolled in the Innovator program. The research questions include: (a) Are there demographic differences among Wellness Innovators who are engaged or disengaged in the Wellness Innovator program?; and (b) Are there perceived differences in direct manager support among Wellness Innovators that are engaged or disengaged in the Wellness Innovator program? These are important questions to ask to help practitioners lead wellness teams that effectively accomplish their roles and responsibilities. In the survey, questions such as "How engaged are you in communicating wellness information, activities, resources, and events with your department/colleagues?" "What factors influence your decision to remain engaged in the Wellness Innovator program?" "What is/was your biggest barrier to facilitating wellness in your department?"; demographic information is being collected to understand these dedicated individuals more fully. In addition, assessments are being gathered to determine the impact of the Innovators on their colleagues' healthy lifestyle beliefs and behaviors. Results will be disseminated once the data are analyzed.

\section{LESSONS LEARNED AND IMPLICATIONS FOR FUTURE PROGRAMMING}

Within four years, the Wellness Innovator program at The Ohio State University has recruited and retained 464 participants. Several lessons have been learned along the way, as well as implications, not only for the Ohio State Wellness Innovator program, but for other champion programs in the field of worksite health promotion.

Wellness champions have a vested interest in achieving personal health and well-being as well as encouraging their colleagues to be healthier and well. Volunteering for this role affords them opportunities to impact their personal wellness and the wellness of their colleagues. The Innovators believe in the purpose and goals of the University employee wellness program, and that they are a critical component to enhance and sustain it. The program facilitators continuously remind Innovators they are crucial to this important university-wide initiative.

In the study, which is currently being implemented, the program facilitators hypothesize support from the Innovator's direct supervisor is one of the most important factors in level of engagement and length of participation in the program. Supervisor support, in the sense of time, resources, and physical space, is quintessential for Innovators to fulfill their role since insufficient support is likely to cause them to be less engaged in the program. Additionally, awareness of the leadership organizational support keeps Innovators involved. The University President, Provost, and CEO of the Academic Medical Center demonstrate an unwavering commitment to the wellness program.

Since this is a volunteer role, it is necessary to provide a booster every so often. For the Innovator program, the booster is in the form of the biannual luncheon, where Innovators come together for two hours to discuss successes and opportunities, and to be reminded of the importance of their role from the University Chief Wellness Officer. They leave feeling reinvigorated.

The strategy session is of upmost importance, from the initial one to the ongoing ones. The initial strategy session sets the agenda, outlining what activities Innovators should implement over a 12-month course. Annual strategy sessions keep them aligned to the plan, on track in the program and engaged in their role.

Regular and consistent communication is important. Since Innovators still have to fulfill their primary job responsibilities, a monthly email and monthly newsletter is used to communicate and disseminate necessary information. The flow of information is dynamic and useful for all involved.

Recognition and sharing success stories is vital to the Innovator program. It allows Innovators to learn new ideas they can promote in their own departments, and recognizes Innovators who have gone above and beyond to promote wellness amongst their colleagues.

Lastly, it is important to get feedback from the Innovators. Perceptions on the program as a whole, including successes and areas of opportunity the facilitators need to consider, are crucial to the quality of the program. Feedback may also come in the form of an Innovator having to discontinue their involvement in the program. The program facilitators understand and respect this decision. Once an Innovator resigns, the facilitator inquiries about a different person who can potentially assume the role, and assist in making the necessary adjustments. It is a volunteer role, and work responsibilities can be a barrier to program engagement. The importance of the dual role of worker/volunteer must be acknowledged in all wellness champion programs. 


\section{CONCLUSIONS}

This paper adds value to the literature in that it described specific components of a wellness champion program, which is important so that this type of program can be scaled to other organizations. Although the program has yet to be determined successful in impacting specific health and wellness outcomes, the program facilitators are able to articulate how the program is making a positive impact on the culture of wellness at Ohio State University. Culture change takes time, often years, especially at large institutions. Continuous quality improvement is needed for a program of this magnitude and importance in order to demonstrate its impact on outcomes and refine it for its greatest impact. 


\section{REFERENCES}

Allen, J.D., Stoddard, A.M., Mays, J., \& Sorensen, G. (2001). Promoting breast and cervical cancer screening at the workplace: results from the Woman to Woman Study. American Journal of Public Health, 91, 584-590.

https://doi.org/10.2105/AJPH.91.4.584

American Heart Association (2015). Healthy Workplace Food and Beverage Toolkit. Retrieved from http://www.heart.org/ HEARTORG/HealthyLiving/WorkplaceHealth/EmployerResources/Healthy-Workplace-Food-and-BeverageToolkit_UCM_465195_Article.jsp

Aoun, S., Sainsbury, K., Mullan, B., \& Shahid, S. (2017). "Champion" behavior in a community obesity reduction program: feedback from peers. Journal of Health Psychology, 22(2), 148-157. https://doi.org/10.1177/1359105315596372

Bondy, S.J., \& Bercovitz, K.L. (2013). "Hike up yer skirt, and quit.” What motivates and supports smoking cessation in builders and renovators. International Journal of Environment Research and Public Health, 10, 623-637. https://doi.org/10.3390/ijerph10020623

Borah, B.J., Egginton, J.S., \& Shah, N.D. (2015). Association of worksite wellness center attendance with weight loss and health care cost savings: Mayo Clinic's experience. Journal of Occupational and Environmental Medicine, 57(3), 229-234. https:// doi.org/10.1097/JOM.0000000000000392

Christakis, N.A., \& Fowler J.H. (2007). The spread of obesity in a large social network over 32 years. New England Journal of Medicine, 357(4), 370-379. https://doi.org/10.1056/NEJMsa066082

Claxton, G., Rae, M., \& Panchal, N. (2011). Employer Health Benefits: Annual Survey. Menlo Park, CA: Henry J. Kaiser Family Foundation and Health Research \& Educational Trust. Retrieved from: https://kaiserfamilyfoundation.

Escoffery, G., Kegler, M.G., \& Alcantara, I. (2011). A qualitative examination of the role of small, rural worksites in obesity prevention. Preventing Chronic Disease, 8(4), A75. http://www.cdc.gov/pcd/issues/2011/jul/10_0185.htm. Accessed March 5, 2017.

James, J. (2013). Health policy brief: Workplace wellness programs. Health Affairs, 2013,1-6.

Leahey, T., \& Wing, R.R. (2012). A randomized controlled pilot study testing three types of health coaches for obesity treatment: professional, peer, and mentor. Obesity, 1(5), 928-934. https://doi.org/10.1002/oby.20271

Linnan, L., Fisher, E., \& Hood, S. (2013). The Power and Potential of Peer Support in Workplace Interventions. American Journal of Health Promotion, 2-10.

Locock, L., Dopson, S., Chambers, D., \& Gabbay, J. (2001). Understanding the role of opinion leaders in improving clinical effectiveness. Social Sciences and Medicine, 53(6), 745-757. https://doi.org/10.1016/S0277-9536(00)00387-7

McPherson, S.L., Joseph, D., \& Sullivan, E. (2004). The benefits of peer support with diabetes. Nursing Forum, 39(4), 5-12. https://doi.org/10.1111/j.1744-6198.2004.tb00009.x

Melnyk, B.M., Amaya, M, Szakacha, L.A., \& Hoying, J. (2016). Relationships Among Perceived Wellness Culture, Healthy Lifestyle Beliefs, and Healthy Behaviors in University Faculty and Staff: Implications for Practice and Future Research. Western Journal of Nursing Research, 38(3), 308-324. https://doi.org/10.1177/0193945915615238

Spoonheim, J. B., \& Pronk, N. P. (2016). Wellness Champion Networks; A Best Practice Resource for Workplace Wellness Programs. ACSM's Health and Fitness Journal, 36- 39.

The Corporate Athlete Course ${ }^{\circledR}$ (2011). Human Performance Institute. Orlando, FL.

Webb, G., Shakeshaft, A., Sanson-Fisher, R., \& Harvard, A. (2009). A systematic review of work-place interventions for alcohol-related problems. Addiction. 104, 365-377. https://doi.org/10.1111/j.1360-0443.2008.02472.x

Wieneke, K., Clark, M., \& Olsen, K. (2016). Development and Impact of a Worksite Wellness Champions Program. American Journal of Health Behavior, 40(2), 215-220. https://doi.org/10.5993/AJHB.40.2.6 
Please address author correspondence to:

Megan Amaya, PhD

Director, Health Promotion \& Wellness; Assistant Professor of Clinical Practice

The Ohio State University

Office of the Chief Wellness Officer

148 Newton Hall | 1585 Neil Avenue Columbus, OH 43210

amaya.13@osu.edu

\section{Components of the Buckeve Wellness Innovators (BWI) Program at}

\section{The Ohio State University}

Recruitment of Innovators

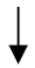

BWI Orientation Session

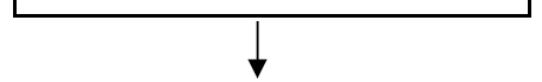

Strategy session with Innovator and supervisor

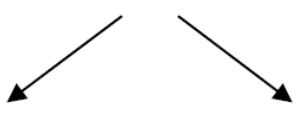

Biannual Innovator webinars

Biannual Innovator luncheons
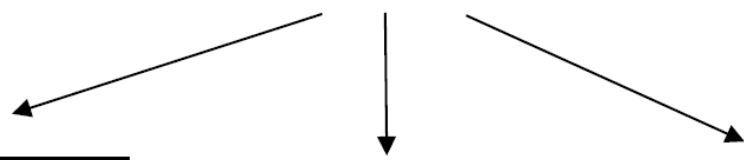

Communicate and participate in universitysponsored wellness programs
Encourage and motivate colleagues to participate in university-sponsored wellness programs and/or healthy behaviors

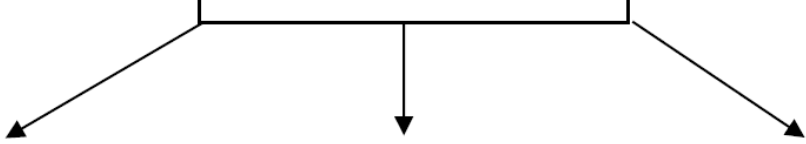

Annual strategy session with Innovator and supervisor
Complete annual quality survey/IRB approved survey to assess impact
Plan wellness activities for department

Apply for Innovator grant funding for unit/department

Figure 1. Innovator on-boarding and roles/responsibilities. 